NYU-TH-07/03/16

\title{
Cargèse Lectures on Brane Induced Gravity
}

\author{
Gregory Gabadadze \\ Center for Cosmology and Particle Physics \\ Department of Physics, New York University, New York, NY, 10003, USA
}

\begin{abstract}
A brief introduction is given to the subject of brane induced gravity. The $5 \mathrm{D}$ example is discussed in detail. The $4 \mathrm{D}$ laws of gravity are obtained on a brane embedded in an infinite volume extra space, where the problem of stabilization of the volume modulus is absent. The theory has two classically disjoint branches of solutions - the conventional and self-accelerated one. The conventional branch gives a perturbatively stable model of a metastable graviton, with potentially testable predictions within the Solar system. The self-accelerated branch, on the other hand, provides an existence proof for an idea that the accelerated expansion of the Universe could be due to modified gravity. The issue of perturbative stability of the self-accelerated branch is obscured by a breakdown of the conventional perturbative expansion. However, a certain exact non-perturbative solution found in hep-th/0612016 exhibits a net negative gravitational mass, while this mass is positive on the conventional branch. This suggest that the self-accelerated solution must be non-perturbatively unstable. A proposal to overcome this problem in an extension of the original model, that also allows for the quantum gravity scale to be unrestricted, is briefly discussed.
\end{abstract}




\section{Introduction}

The discovery of Refs. [1] that that the present-day expansion of the Universe is accelerating, has been confirmed by a number of subsequent efforts. One way to parametrize the accelerated expansion is to postulate the existence of a "dark energy" component in the Einstein equation:

$$
G_{\mu \nu}=8 \pi G_{N}\left(T_{\mu \nu}^{\text {matter }}+T_{\mu \nu}^{\text {dark energy }}\right),
$$

were $G_{\mu \nu}=R_{\mu \nu}-g_{\mu \nu} R / 2$, is the $4 \mathrm{D}$ Einstein tensor of the metric $g_{\mu \nu}(x)$, and

$T_{\mu \nu}^{\text {matter }}$ and $T_{\mu \nu}^{\text {dark energy }}$ denote the stress-tensors for matter (including dark matter) and dark energy, respectively; the latter has to have a negative enough pressure to account for the observations.

On the other hand, one can consider another logical possibility that the accelerated expansion is due to modified General Relativity (GR). Schematically, the modified Einstein equation could be written as:

$$
G_{\mu \nu}-\mathcal{K}_{\mu \nu}\left(g, m_{c}\right)=8 \pi G_{N} T_{\mu \nu}^{\text {matter }},
$$

where $\mathcal{K}_{\mu \nu}\left(g, m_{c}\right)$ denotes a tensor that could depend on a metric $g$, its derivatives, as well as on other fields not present in GR. Moreover, $\mathcal{K}$ contains a dimensionful constant $m_{c} \sim H_{0} \sim 10^{-42} \mathrm{GeV}$, which sets a distance/time scale $r_{c} \equiv m_{c}^{-1}$ at which the Newtonian potential obtained from (2) significantly deviates from the conventional one. One concrete example of $\mathcal{K}_{\mu \nu}\left(g, m_{c}\right)$ is given by the DGP model [2], and will be discussed in detail in the next section.

At a first sight, there does not seem to be a difference between (2) and (11), as the new term on the l.h.s. of (2) could be transfered to its r.h.s. and regarded as the "dark energy" component, similar to the one present in (1).

In reality, however, the difference between (22) and (1) is significant. It is typically implied in (11) that the dark energy is either due to cosmological constant or a light scalar field. This field forms an independent sector of the theory, the dynamics of which is not restricted by severe constraints that general covariance imposes on tensor fields. In contrast with this, the new term in (2) contains metric itself in a nontrivial way. As such it is highly restricted by general covariance. This gives rise to both the theoretical and observational differences between models of "dark energy" and modified gravity.

It is worth emphasizing that a primary theoretical motivation for the models of modified gravity is to evade the S.Weinberg's no-go theorem on the "old" cosmological constant problem (CCP) [3], see Ref. [4] for a summary of these discussions. This attractive possibility still exists, in principle, in models of brane induced gravity with the number of space-time dimensions $D \geq 6$ [5, 6, 7], however, many aspects of those models are not well-understood, and we won't be discussing them here. Instead we concentrate on the 5D brane induced gravity [2]. The latter does not offer a solution to the old CCP, however, it can be used as an example for understanding of a new dynamics introduced by modified gravity. 


\section{Brane induced gravity}

An explicit example of the modified Einstein equation (22) is provided by the DGP model [2]. All the known 4D interactions, except gravity, are thought to be confined to a brane that is embedded in a $5 \mathrm{D}$ infinite-volume (uncompactified) empty space where only gravity propagates. In this setup, the additional term on the l.h.s. of (2) is provided by the $4 \mathrm{D}$ extrinsic curvature terms of the brane

$$
G_{\mu \nu}-m_{c}\left(K_{\mu \nu}-g_{\mu \nu} K\right)=8 \pi G_{N} T_{\mu \nu}(x) .
$$

Here, $G_{\mu \nu}=R_{\mu \nu}-g_{\mu \nu} R / 2$, is the $4 \mathrm{D}$ Einstein tensor for the metric that depends on both $4 \mathrm{D}$ coordinates $x_{\mu}$, and the fifth coordinate $y, g_{\mu \nu}(x, y) ; K=g^{\mu \nu} K_{\mu \nu}$, is the trace of the extrinsic curvature tensor

$$
K_{\mu \nu}=\frac{1}{2 N}\left(\partial_{y} g_{\mu \nu}-\nabla_{\mu} N_{\nu}-\nabla_{\nu} N_{\mu}\right),
$$

and $\nabla_{\mu}$ is a $4 \mathrm{D}$ covariant derivative w.r.t. the metric $g_{\mu \nu}(x, y)$. We introduced the lapse scalar field $N$, and the shift vector field $N_{\mu}$ [8]:

$$
g_{\mu 5} \equiv N_{\mu}=g_{\mu \nu} N^{\nu}, \quad g_{55} \equiv N^{2}+g_{\mu \nu} N^{\mu} N^{\nu} .
$$

In the DGP model, equation (3) is accompanied by the $\{\mu \nu\}$ equation in the bulk, and the $\{\mu 5\}$ and $\{55\}$ equations which respectively read

$$
\begin{array}{r}
G_{\mu \nu}^{(5)}=0 \quad \text { for } \quad y \neq 0, \\
\nabla^{\mu} K_{\mu \nu}=\nabla_{\nu} K, \\
R=K^{2}-K_{\mu \nu} K^{\mu \nu} .
\end{array}
$$

Here $G_{\mu \nu}^{(5)}$ denotes the 5D Einstein tensor for the 5D metric $g_{A B}(x, y)(\mathrm{A}, \mathrm{B}=0,1,2,3,5)$, and $g_{\mu \nu}(x, y)$ is its $4 \mathrm{D}$ part. Note that the $\{\mu 5\}$ and $\{55\}$ equations, (7) and (8), should be satisfied in the bulk, $y \neq 0$, as well as on the brane, $y=0$.

The above set of equations can be derived by the variational principle using the action [2]

$$
S=\frac{M_{\mathrm{Pl}}^{2}}{2} \int d^{4} x \sqrt{g_{4}} R\left(g_{4}\right)+\frac{M_{*}^{3}}{2} \int d^{4} x d y \sqrt{g_{5}} \mathcal{R}\left(g_{5}\right)+\text { surface terms },
$$

where $g_{4 \mu \nu} \equiv g_{\mu \nu}(x, 0)$, and $g_{5}$ refers to the full 5D metric; $R$ and $\mathcal{R}$ are the fourdimensional and five-dimensional Ricci scalars respectively, and $M_{*}$, stands for the fundamental gravitational scale of the bulk theory. The brane is located at $y=0$ and $\mathbf{Z}_{2}$ symmetry across the brane is imposed. The boundary Gibbons-Hawking term should be taken into account to warrant the correct Einstein equations in the bulk. The matter fields, that are also omitted here for simplicity, are assumed to be localized on the brane. 
Another form of the action (9) can be given in the ADM formalism

$$
S=\frac{M_{\mathrm{Pl}}^{2}}{2} \int d^{4} x \sqrt{g_{4}} R\left(g_{4}\right)+\frac{M_{*}^{3}}{2} \int d^{4} x d y \sqrt{g} N\left(R+K^{2}-K_{\mu \nu}^{2}\right) .
$$

The above action gives rise to the equations of motion (3,6 6 , 8 ), where

$$
m_{c} \equiv \frac{2 M_{*}^{3}}{M_{\mathrm{Pl}}^{2}} .
$$

In order for $m_{c}$ to be of order $H_{0}$, we need that the bulk quantum gravity scale to be $M_{*} \sim 100 \mathrm{MeV}$. Such a low value of the quantum gravity scale is in no conflict with observations, however, it presents an obstacle for a string theory realization of the above model. In the next section, we will show how a mild extension of the model can relax the constraint on $M_{*}$, in which case it could take an arbitrary value below the 4D Planck mass [9].

\section{Role of non-linearities}

To better understand the linearized theory of (9), it is instructive to consider an analogous scalar model [2]

$$
-\frac{M_{\mathrm{Pl}}^{2}}{2} \int d^{4} x\left(\partial_{\mu} \phi(x, 0)\right)^{2}-\frac{M_{*}^{3}}{2} \int d^{4} x d y\left(\partial_{A} \phi(x, y)\right)^{2},
$$

where the dimensionless scalar field $\phi$ is to mimic the 5D graviton of the previous section. We impose the $\mathbf{Z}_{\mathbf{2}}$ symmetry across the $y=0$ boundary, and add to the above action the coupling of $\phi$ to a source $J$, also localized on the brane, $\int d^{4} x d y \delta(y) J \phi$. To obtain the junction condition, we integrate the equation of motion obtained from (12) w.r.t. $y$, from $0^{-}$to $0^{+}$. The resulting equation written at $y=0^{+}$reads:

$$
-\left.\partial_{\mu}^{2} \phi\right|_{y=0}-\left.m_{c} \partial_{y} \phi\right|_{y=0^{+}}=J / M_{\mathrm{Pl}}^{2} \text {. }
$$

The bulk equation is nothing but a 5D free scalar equation

$$
\partial^{A} \partial_{A} \phi=0 \text { for } y \neq 0 \text {. }
$$

It is straightforward to find a solution to the bulk and junction equations. For this we turn to the momentum space w.r.t. the $4 \mathrm{D}$ coordinates while keeping the fifth dimension intact. The (decaying in the bulk) solution reads:

$$
\tilde{\phi}(p, y)=\left(\frac{\tilde{J}}{M_{\mathrm{Pl}}^{2}}\right) \frac{\exp (-p|y|)}{p^{2}+m_{c} p},
$$

where the sign "tilde" denotes the Fourier transformed quantities, and we introduced the Euclidean 4-momentum square as follows: $p^{2} \equiv p^{\mu} p_{\mu}=-p_{0}^{2}+p_{1}^{2}+p_{2}^{2}+p_{3}^{2} \equiv$ $p_{4}^{2}+p_{1}^{2}+p_{2}^{2}+p_{3}^{2}$, and set the notation $p \equiv \sqrt{p^{2}}$. 
The solution (15) exhibits a number of interesting properties:

(1) At short distances (i.e., the large momenta $p \gg m_{c}$ ) it reduces to a $4 \mathrm{D}$ solution with the inverse square law, $1 / r^{2}$, for the force mediated by this scalar. At large distances (i.e., small momenta $p \ll m_{c}$ ) it turns into a 5D solution with the $1 / r^{3}$ force law.

(2) The expression (15) appears to have two poles, one at $p^{2}=0$ and another one at $p=\sqrt{p^{2}}=-m_{c}$. However, the residue of the former pole is zero, consistent with the expectation that there is no normalizable $4 \mathrm{D}$ massless state in the spectrum.

The second pole appears on a non-physical Riemann sheet of the complex $p^{2}$ plane (see details in Ref. [4]), and describes a resonance-like state. The branch-cut on the complex $p^{2}$-plane is due to the $\sqrt{p^{2}}$ term in (15), and reflects the presence of a continuum of the Kaluza-Klein (KK) states.

(3) Two localized sources on a brane exchange one 5D scalar state the propagator of which can be read off (15). From the 4D perspective what is being exchanged is an infinite number of $\mathrm{KK}$ states, the $4 \mathrm{D}$ couplings of which are suppressed as compared to the ordinary $5 \mathrm{D}$ theory by the following factor [10]:

$$
\frac{1}{1+\left(m^{2} / m_{c}^{2}\right)}
$$

where $m$ denotes the mass of a given KK state. Therefore, the heavier the KK state the more its suppression. That is why at distances $\lesssim r_{c}=m_{c}^{-1}$ one recovers $4 \mathrm{D}$ Newtonian potential.

A similar calculation can be performed for the linearized gravitational theory [2]. The object of the primary interests here is the one-graviton exchange amplitude between two sources. The expression for the resulting amplitude is gauge independent and reads as follows:

$$
\mathcal{A}_{1 \text {-graviton }}(p, y)=\frac{T_{1 / 3}^{2}}{p^{2}+m_{c} p} \exp (-p|y|),
$$

where

$$
T_{1 / 3}^{2} \equiv 8 \pi G_{N}\left(T_{\mu \nu}^{2}-\frac{1}{3} T \cdot T\right)
$$

As in the scalar example, the pole at $p^{2}=0$ has zero residue, and the second pole in (17) is on a non-physical Riemann sheet. Therefore, the amplitude $\mathcal{A}_{1 \text {-graviton }}$ describes propagation of a metastable state with the lifetime $\sim m_{c}^{-1}$, which decays into a continuum of the KK modes.

However, there is a crucial difference from the scalar case. This has to do with the numerator of (17). In the limit $m_{c} \rightarrow 0$ the numerator does not reduce to the analogous expression in GR. The latter takes the form:

$$
8 \pi G_{N}\left(T_{\mu \nu}^{2}-\frac{1}{2} T \cdot T\right)
$$


The difference between (18) and (19) is due to the fact that a 5D graviton (or a massive graviton for that matter) propagates 5 on-shell degrees of freedom (helicity-2, helicity-1, and helicity-0), while the GR graviton propagates only 2 on-shell degrees of freedom (helicity-2 state). The helicity-1 state of the 5D graviton does not contribute to the one-graviton exchange amplitude (18) because of the conservation of the stress-tensor. However, the helicity-0 state does contribute and gives rise to the finite difference between (18) and (19). Observationally (18) is excluded! This is the essence of the van Dam-Veltman-Zakharov discontinuity (vDVZ) [11.

Fortunately, the vDVZ argument fails for observationally interesting sources. This is due to nonlinear interactions [12, 13], and can easily be understood as follows [13]: the longitudinal part of the graviton propagator in DGP contains terms proportional to

$$
\frac{p_{\mu} p_{\nu}}{m_{c} p}
$$

This term does not contribute to the amplitude $\mathcal{A}_{1 \text {-graviton }}$ because of conservation of the stress-tensor, however, it does contribute already in the first nonlinear correction (since the stress-tensor is only covariantly conserved in the non-linear theory). Due to the singularity in $m_{c}$ in (20), the perturbation theory breaks down precociously. However, this breakdown is an artifact of an ill-defined perturbative expansion - the known exact solutions of the model have no trace of breaking [13]. The perturbative expansion in powers of $G_{N}$ gets "contaminated" by another dimensionful parameter $1 / m_{c}$, and this leads to its breakdown.

Under the circumstances, one could either adopt a different type of expansion, e.g., an expansion in the small parameter $m_{c}$ [13, 14], or look at exact solutions 1. Both of these programs have been carried out to a certain extent. Here we summarize the main results.

The model has one adjustable parameter - the distance/time scale $r_{c}=m_{c}^{-1}$. Distributions of matter and radiation which are homogeneous and isotropic at scales $\gtrsim r_{c}$ exhibit the following properties: for distance/time scales $\ll r_{c}$ the solutions approximate General Relativity (GR) to a high degree of accuracy, while for scales $\gtrsim$ $r_{c}$ they dramatically differ [19, 20, 13]. Since $r_{c}^{-1} \sim H_{0} \sim 10^{-42} \mathrm{GeV}$, the deviations from GR could lead to observational consequences in late-time cosmology, see, e.g., [20], [21]- 30].

On the other hand, sources of matter and radiation with typical inhomogeneity scales less than $r_{c}$ have somewhat different properties. These are easier to discuss for a Schwarzschild source - a spherically-symmetric distribution of matter of the mass $M$ and radius $r_{0}$, such that $r_{M}<r_{0} \ll r_{c}\left(r_{M} \equiv 2 G_{N} M\right.$ is the Schwarzschild radius). For such a source a new scale, that is a combinations of $r_{c}$ and $r_{M}$, emerges

\footnotetext{
${ }^{1}$ It is also possible to modify the theory at the linearized level so that the conventional perturbative expansion is well-behaved [15, 16, , 17, , 18].
} 
(the so-called Vainshtein scale²) [13]:

$$
r_{*} \equiv\left(r_{M} r_{c}^{2}\right)^{1 / 3}
$$

Below this scale the predictions of the theory are in a good agreement with the GR results. Above this scale, however, gravity of a compact object deviates substantially from the GR result. Note that $r_{*}$ is huge for typical astrophysical objects. An isolated star of a solar mass would have $r_{*} \sim 100 p c$. However, if we draw a sphere of a $100 p c$ radius with the Sun in its center there will be many other starts enclosed by that sphere. The matter enclosed by this sphere would have even larger $r_{*}$. We could draw a bigger sphere, but it will enclose more matter which would yield yet larger $r_{*}$ and so on. An isolated object which could potentially be separated from a neighboring one by a distance larger than its own $r_{*}$ is a cluster of galaxies. For typical clusters, $r_{*} \sim($ few $M p c)$ is just somewhat larger than their size.

The above arguments suggest that interactions of isolated clusters may be different in the DGP model. On the other hand, at scales beneath a few Mpc or so, there will be agreement with the GR results with potentially interesting small deviations.

For simplicity, we discuss below these issues for a single isolated Schwarzschild source. There exist in the literature two different (but both partial) solutions for the Schwarzschild problem in the DGP model. The first one is based on approximate expansions in the $r \ll r_{*}$ and $r \gg r_{*}$ regions [2, 13, 14] (see also [31]). We call this set of results the perturbative Schwarzschild (PS) solution. The second one [32] is a solution on the brane that interpolates smoothly from $r \ll r_{*}$ to $r \gg r_{c} \gg r_{*}$, and is non-analytic in the either parameters used to obtain the PS solution. We call this the non-perturbative Schwarzschild (NPS) solution. What is certain, is that at observable distances both solutions are in good agreement with the GR results, but predict a tiny and potentially measurable deviations from GR [33, 34] (see also [35, 36]).

It is important to understand which of these two solutions, if any, is physically viable. Since neither of the two were solved completely in the entire 5D space-time, a first step to discriminate between them would be to look closely at the predictions that could by tested observationally. This was discussed in detail in Ref. [36]. We briefly summarize some of the results.

Let us start with the Newton potential $\varphi(r)$. The result for $r \ll r_{*}$ leads:

$$
-2 \varphi=\frac{r_{M}}{r}-\alpha m_{c}^{2} r^{2}\left(\frac{r_{*}}{r}\right)^{\frac{3}{2}-\beta}+\ldots
$$

where $\alpha= \pm \sqrt{2}$ and $\beta=0$ for the perturbative solution (PS), while $\alpha= \pm 0.84$ and $\beta=3 / 2-2(\sqrt{3}-1) \simeq 0.04$ for the non-perturbative solution (NPS) [32] (two signs for $\alpha$ correspond to the tow different branches of solutions, see discussions below).

\footnotetext{
${ }^{2}$ A similar, but not exactly the same scale was discovered by Vainshtein in massive gravity [12, hence the name.
} 
The deviation from $4 \mathrm{D}$ gravity at $r \ll r_{*}$ gives rise to the additional perihelion precession of circular orbits [33, 34] (see also [35] for comprehensive studies of these and related issues). In a simplest approximation this effect is quantified by a fraction of the deviation of the potential $\varphi$ from its Newtonian form

$$
\epsilon \equiv \frac{\Delta \varphi}{\varphi}
$$

This can be used to evaluate an additional perihelion precession of orbits in the Solar system [33, 34] $]^{3}$. The $\epsilon$ ratio is somewhat different for the non-perturbative solution (NPS) as compared to the perturbative solution (PS) used in Refs. [33, 34. This difference has been calculated [36]:

$$
\frac{\epsilon_{N P S}}{\epsilon_{P S}} \simeq 0.59\left(\frac{r}{r_{*}}\right)^{0.04}
$$

The perihelion precession per orbit is

$$
\Delta \varphi=2 \pi+\frac{3 \pi r_{M}}{r} \mp \frac{3 \pi|\alpha|}{4}\left(\frac{r}{r_{*}}\right)^{3 / 2}\left(\frac{r}{r_{*}}\right)^{0.04} .
$$

The second term on the RHS is the Einstein precession, and the last term arises due to modification of gravity. For the PS this was first calculated in Refs. [33, 34] ; the solution (25) is written for the NPS and is somewhat different.

For the Earth-Moon system $r \simeq 3.84 \times 10^{10} \mathrm{~cm}$ and $r_{*}^{\text {Earth }} \simeq 6.59 \times 10^{12} \mathrm{~cm}$; as a result the ratio in (24) is approximately 0.48 . Therefore, the predictions of the non-perturbative solution for the additional perihelion precession of the Moon is a factor of two smaller than the predictions of the perturbative solution. The result of (25) for the additional precession (the last term on the RHS) is $\mp 0.7 \times 10^{-12}$ (the plus sign for the self-accelerated branch). This is below the current accuracy of $2.4 \times 10^{-11}$ [37], but could potentially be probed in the near future [38].

A similar calculations can be performed for the anomalous Martian precession [33, 34]. For the Sun-Mars system we use $r_{\text {Sun-Mars }}=2.28 \times 10^{13} \mathrm{~cm}$ and $r_{*}^{\text {Sun }}=$ $4.9 \times 10^{20} \mathrm{~cm}$. The additional precession of the Mars orbit is $\sim \mp 1.3 \times 10^{-11}$, which should be contrasted with a potential accuracy of the Pathfinder mission $\sim 9 \times 10^{-11}$.

\section{The quantum gravity scale}

We have discussed in the previous section that the value of the 5D Planck mass $M_{*}$ is restricted by the requirement that $m_{c}=2 M_{*}^{3} / M_{\mathrm{Pl}}^{2}$ be of order $H_{0}$, leading to the value of the bulk "quantum gravity" scale $M_{*} \sim 100 \mathrm{MeV}$.

\footnotetext{
${ }^{3}$ Note that in the leading order of the relativistic expansion the answer is given by the correction to the Newtonian potential, while the correction to the $r r$ component of the metric is not important.
} 
Here we discuss a slight modification of the DGP model which retains the most of the important properties of the original theory and yet allows to relax the constrain on the bulk quantum gravity scale $M_{*}$.

For simplicity we first discuss this for the scaler example. The main idea is that the strength of the 5D kinetic term could depend on the $y$ coordinate, so that its value is small on the brane but is large off the brane. We could parametrize this as

$$
\int d x d y F(x, y)\left(\partial_{A} \phi(x, y)\right)^{2}
$$

where the new scalar $F$ (a "dilaton") is assumed to have an $x$ independent profile in the $y$ direction such that $F(y \rightarrow 0)=m_{c} M_{\mathrm{Pl}}^{2} / 4$, while $F(y \neq 0)$ sets the bulk quantum gravity scale, which is unrestricted and could be as large as $M_{\mathrm{Pl}}$.

Let us perform these calculations more carefully. To account for the above properties, we introduce an additional term into the action (12) which is just a opposite sign 5D kinetic term peaked on the brane. To make things tractable, we smear the brane, that is, instead of the Dirac function $\delta(y)$, we use its regularized version $\delta(y) \rightarrow \bar{\delta}(y) \equiv \pi^{-1} \varepsilon /\left(y^{2}+\varepsilon^{2}\right)$, with $\varepsilon \rightarrow 0$. The term that we'll be adding to (12) then reads:

$$
\frac{M^{2}}{2} \int d x d y \bar{\delta}(y)\left(\partial_{A} \phi(x, y)\right)^{2}
$$

With this term included the variation of the action $\delta S=0$ with the appropriate boundary conditions gives:

$$
-\left(M_{\mathrm{Pl}}^{2}-M^{2}\right) \bar{\delta}(y) \partial_{\mu}^{2} \phi-M_{*}^{3} \partial_{\mu}^{2} \phi-\partial_{y}\left(\left(M_{*}^{3}-M^{2} \bar{\delta}(y)\right) \partial_{y} \phi\right)=J \bar{\delta}(y) .
$$

Next we take the integral of both sides of this equation w.r.t. $y$ from $-\varepsilon$ to $+\varepsilon$, and then turn to the limit

$$
M \rightarrow 0, \quad \varepsilon \rightarrow 0, \quad M^{2} / \varepsilon \sim M^{2} \bar{\delta}(0) \equiv \bar{M}^{3}>M_{*}^{3},
$$

where we keep $\bar{M}$ fixed, and its value somewhat lower than $M_{*}$. The resulting equation reads:

$$
-\partial_{\mu}^{2} \phi-\frac{2\left(M_{*}^{3}-\bar{M}^{3}\right)}{M_{\mathrm{Pl}}^{2}} \partial_{y} \phi=J / M_{\mathrm{Pl}}^{2} .
$$

Finally, introducing

$$
m_{c} \equiv \frac{2\left(M_{*}^{3}-\bar{M}^{3}\right)}{M_{\mathrm{Pl}}^{2}},
$$

where the positive numerical value of $m_{c}$ will be tuned to the Hubble scale today $m_{c} \sim H_{0} \sim 10^{-42} \mathrm{GeV}$, we get the desired junction conditions

$$
-\partial_{\mu}^{2} \phi-m_{c} \partial_{y} \phi=J / M_{\mathrm{Pl}}^{2}
$$


Two comments. First, the wrong-sign kinetic term (27) that is peaked only on the brane is dominated by the large positive 4D kinetic term in (12), proportional to $M_{\mathrm{Pl}}^{2}>M^{2}$. Second, the number of adjusted parameters here is the same as in DGP: In (12) one should tune the value of $M_{*}$ such that the ratio $2 M_{*}^{3} / M_{\mathrm{Pl}}^{2}$ is of order $H_{0}$, while in the action (27) the value of $M_{*}<M_{\mathrm{Pl}}$ can be arbitrary, as long as one tunes the value of $\bar{M}$ so that (31) is of order $H_{0}$.

In the case of gravity, to which we turn now, similar considerations can applied. As before, we smooth out the brane by replacing $\delta(y) \rightarrow \bar{\delta}(y)$, and think of the 5D EH term to have a profile due to the "dilaton" field

$$
\int d^{4} x d y \sqrt{g_{5}} F(x, y) \mathcal{R}\left(g_{5}\right)
$$

such that the 5D gravitational coupling on the brane is strong, while it becomes weak in the bulk.

The above construction could be parametrized by adding the following boundary (worldvolume) term to the DGP action:

$$
-\frac{M^{2}}{2} \int d^{4} x d y \bar{\delta}(y) \sqrt{g_{5}} \mathcal{R}\left(g_{5}\right)=-\frac{M^{2}}{2} \int d^{4} x d y \bar{\delta}(y) \sqrt{g} N\left(R+K^{2}-K_{\mu \nu}^{2}\right)
$$

where the r.h.s. of (34) is obtained by using the standard ADM decomposition. The total action in the ADM formalism reads:

$$
\begin{array}{r}
S_{\mathrm{mod}}=\frac{M_{\mathrm{Pl}}^{2}}{2} \int d^{4} x \sqrt{g_{4}} \\
R\left(g_{4}\right)+\frac{M_{*}^{3}}{2} \int d^{4} x d y \sqrt{g} N\left(R+K^{2}-K_{\mu \nu}^{2}\right) \\
-\frac{M^{2}}{2} \int d^{4} x d y \bar{\delta}(y) \sqrt{g} N\left(R+K^{2}-K_{\mu \nu}^{2}\right)
\end{array}
$$

where it is implied that the $4 \mathrm{D}$ EH term is also smeared over the same scale as the $5 \mathrm{D}$ term 4 . The equations of motion are straightforward to derive from (35). The $\{\mu \nu\}$ equation in the bulk, and $\{\mu 5\}$ and $\{55\}$ equations read as follows:

$$
\begin{aligned}
G_{\mu \nu}^{(5)}=0 \quad \text { for }|y| & >\epsilon, \\
\left(M_{*}^{3}-M^{2} \bar{\delta}(y)\right)\left(\nabla^{\mu} K_{\mu \nu}-\nabla_{\nu} K\right) & =0 \\
\left(M_{*}^{3}-M^{2} \bar{\delta}(y)\right)\left(R-K^{2}+K_{\mu \nu} K^{\mu \nu}\right) & =0 .
\end{aligned}
$$

As in the scalar case, we will be looking at this theory in the limit (29). The above equations reduce to (6, 6 ).

The Israel junction condition across the brane gets modified because of the new term in (35). In the limit (29) this condition reads:

$$
G_{\mu \nu}-\frac{2\left(M_{*}^{3}-\bar{M}^{3}\right)}{M_{\mathrm{Pl}}^{2}}\left(K_{\mu \nu}-g_{\mu \nu} K\right)=8 \pi G_{N} T_{\mu \nu}(x)
$$

\footnotetext{
${ }^{4}$ For the regularization of $4 \mathrm{D}$ and $5 \mathrm{D}$ EH terms, see, [39] and [17, respectively.
} 
If $\bar{M}=0$, as in (9), we get back the result (3) with $m_{c}=2 M_{*}^{3} / M_{\mathrm{Pl}}^{2}$. However, $\bar{M}$ does not have to be zero. For an arbitrary value of $M_{*}$ we tune the value of $\bar{M}$ so that the crossover scale (31), which appears in (39), is adjusted to the value of the present-day Hubble scale $m_{c} \sim H_{0} \sim 10^{-42} \mathrm{GeV}$. Hence, (39) recovers (46).

What we have shown is that for $M \ll M_{\mathrm{Pl}}$ the junction condition is not modified as compared to DGP. The 5D gravitational constant in the bulk equations of (35) would change, though, if we were to consider sources extending into the bulk. However, our primary interest is in the sources localized on the brane, and for those, the new model (35) with $M \ll M_{\mathrm{Pl}}$. recovers the results of DGP.

\section{Cosmology}

Let us turn to the cosmological solutions. To this end we consider distributions of matter and radiation that are homogeneous at scales $\gg r_{c}$. Therefore, the complications due to non-linear dynamics outlined in the previous section do not apply to these sources 5 .

The metric is parametrized as follows:

$$
d s^{2}=-P^{2}(t, y) d t^{2}+Q^{2}(t, y) \gamma_{i j} d x^{i} d x^{j}+\Sigma^{2}(t, y) d y^{2} .
$$

There are two branches of solutions that are labeled by an integer $\epsilon= \pm 1$ [19]:

$$
P(t, y)=1+\epsilon|y| \frac{\ddot{a}}{\sqrt{\dot{a}^{2}+k}}, \quad Q(t, y)=a(t)+\epsilon|y| \sqrt{\dot{a}^{2}+k}, \quad \Sigma(t, y)=1 .
$$

Here we included a nonzero spatial curvature $k$. With this Ansatz, the Friedmann equation on the brane follows from (3), and can be expressed in terms of the $4 \mathrm{D}$ Hubble parameter $H \equiv \dot{a} / a$. For a simplest case of a brane without matter/radiation on it, and with $k=0$ the equation reads [19]

$$
H^{2}-\epsilon m_{c}|H|=0 .
$$

The case with $\epsilon=+1$ admits a dS solution with $H=m_{c}$. This is called the selfaccelerated solution, as it gives rise to the accelerated expansion of the universe due to modified gravity. We emphasize that the minus sign between the two terms in (42) is guaranteed by the choice of the positive sign in front of the terms in (41) that are proportional to $|y|$. If we were to choose the latter signs to be negative, we would have obtained the Friedmann equation $H^{2}+m_{c}|H|=0$, which does not admit the dS solution. The latter corresponds to the choice $\epsilon=-1$, and is referred to as the conventional branch. There is no acceleration produced by modified gravity on this branch. It has to be obtained, e.g., by introducing a small cosmological constant on the brane. An interesting observation concerning this branch is the following [40]:

\footnotetext{
${ }^{5}$ Although, those complications will be relevant to perturbations about the background cosmological solutions.
} 
the cosmological expansion from a $4 \mathrm{D}$ point of view looks as if it had the equation of state parameter less than -1 . This is because gravity turns into the $5 \mathrm{D}$ regime at scales $H \sim m_{c}$, which implies less "deceleration" of matter due to attractive gravity, and this appears to a 4D observed as "faster" acceleration [40].

It is straightforward to introduce matter/radiation on the brane. Following [19] we obtain the Friedmann equation

$$
H^{2}+\frac{k}{a^{2}}=\left(\sqrt{\frac{8 \pi G_{N}}{3} \rho+\frac{m_{c}^{2}}{4}}+\epsilon \frac{m_{c}}{2}\right)^{2}
$$

which should be amended by the conventional conservation equation for the fluid of density $\rho$ and pressure $p: \dot{\rho}+3 H(\rho+p)=0$. The latter being a result of the matter stress-tensor conservation $\nabla^{\mu} T_{\mu \nu}=0$, which can be verified, e.g., by taking a covariant derivative of both sides of (3) and using (17).

When $H \gg m_{c}$, i.e., in the early universe, eq. (43) reduces to the conventional Friedmann equation. However, at late times, when $H \sim m_{c}$, the cosmological evolution changes. On the conventional branch $(\epsilon=-1)$ it turns into the expansion driven by the 5D force law, and on the self-accelerated branch $(\epsilon=1)$ it turns into the dS-like expansion.

How about perturbations on the cosmological solutions? We start with the conventional branch. Here, the small perturbations about the Minkowski background are stable [2]. However, the conventional perturbative expansion breaks down near realistic sources, as was discussed in the previous section. As a result, the model exhibits the strongly-coupled behavior already at the classical level [13] in the domain where the extrinsic curvature square is $\gtrsim m_{c}^{2}$. The same applies to the selfaccelerated branch. Because of this, the question of stability of the self-accelerated solution [43] - 46], which happens to be in the strongly coupled regime due to its curvature, becomes difficult to address within the perturbative approach [47, 48]6.

Luckily, certain non-linear solutions have been found. This is a case for a Schwarzschild-like solution, for which the 4D metric was exactly obtained [32, and for the Domain Wall solution for which the full 5D metric was found in Ref. [49]. In both cases, the mass (tension) of the solution gets screened by gravitational effects, and these sources on the self-accelerated background look as if they had a negative net 5D mass (tension) 7 . This suggests that the self-accelerated background may not be problem-free in the full non-linear theory.

What is a root-cause of this behavior? The self-accelerated solution exists only for a certain choice of the sign of the extrinsic curvature, and this choice is such that

\footnotetext{
${ }^{6}$ One could look at this breakdown in terms of the scalar "conformal" mode. In massive gravity this mode decouples from the rest of the modes in a certain limit [41. In the DGP model, however, such a decoupling does not take place [42].

${ }^{7}$ In contrast with this, screening of the similar sources on the conventional branch of DGP, leaves them with positive 5D mass (tension) [32, 49.
} 
it requires a growing metric in the bulk. For instance, in a simplest spatially-flat case, the full 5D metric of the self-accelerated solution takes the form [19]:

$$
d s^{2}=(1+H|y|)^{2}\left\{-d t^{2}+e^{2 H t} d \vec{x}^{2}\right\}+d y^{2},
$$

where $y$ is the 5 th coordinate and $H$ denotes the dS expansion rate of the $4 \mathrm{D}$ worldvolume (the latter is labeled here by Cartesian coordinates $(t, \vec{x})$, and we use the $5 \mathrm{D}$ coordinate system in which the brane is located at $y=0$.). The unusual feature of the above metric is that it grows in the bulk, even though the worldvolume metric is that of dS space. A linearly growing metric, similar to (44), would have been produced by a negative tension 3 -bran 8 . The growing metric (44) imprints its "negative" effects on the brane worldvolume through the extrinsic curvature, giving rise to the solutions mentioned in the previous paragraph.

Can this problem be cured? It turns out that one can modify the DGP equations in such a way that the new system admits a background that is equivalent to the self-accelerated solution on the 4D brane, however, differs from it in the bulk.

The new solution that we will discuss takes the form:

$$
d s^{2}=(1-H|y|)^{2}\left\{-d t^{2}+e^{2 H t} d \vec{x}^{2}\right\}+d y^{2} .
$$

In order to obtain this solution one needs to flip the sign in front of the extrinsic curvature term in one of the DGP equations, keeping the rest of the equations intact. Below will discuss how such equations can be obtained by modifying the DGP action.

The solution (45) is formally identical to that for a 3-brane endowed with a positive 4D cosmological constant (brane tension) which is embedded in 5D empty space in 5D GR [50]. However, unlike the latter, the worldvolume expansion in the present case (45) is due to modified gravity, while the $4 \mathrm{D}$ cosmological constant is set to zero. This difference is what is responsible for the modified Friedmann equation, and distinct cosmological evolution on the self-accelerated background.

The bulk space in both (44) and (45) is locally equivalent to 5D Minkowski space. In the chosen coordinate system the solution (45) encounters the Rindler horizon at $|y|=H^{-1}$. However, an analytic continuation beyond this point can be performed by employing new coordinates. In that coordinate system the brane (with closed spatial sections) can be regarded as a $4 \mathrm{D} \mathrm{dS}$ bubble that is first contracting and then re-expanding in 5D Minkowski space.

The proposal of Ref. [9] is to add a new term on the brane worldvolume such that the sign in front of the second term on the l.h.s. of (3) would flip. In other words, we introduce a new set of equations in which (3) is replaced by:

$$
G_{\mu \nu}+m_{c}\left(K_{\mu \nu}-g_{\mu \nu} K\right)+\gamma \Sigma_{\mu \nu}=8 \pi G_{N} T_{\mu \nu}(x),
$$

while all the other equations (6), (17) and (8) remain intact. Here, in a simplest case $\Sigma_{\mu \nu} \equiv\left(G_{\mu \nu}-\frac{1}{2} g_{\mu \nu}\left(K^{2}-K_{\alpha \beta}^{2}\right)-2\left(K_{\mu}^{\alpha} K_{\nu \alpha}-K K_{\mu \nu}\right)\right)$, and the small coefficient $\gamma \lesssim 1$

\footnotetext{
${ }^{8}$ This could be in, e.g., 5D Minkowski or Anti de Sitter (AdS) space-time.
} 
is in general nonzero for a regularized brane width. An important property of the tensor $\Sigma_{\mu \nu}$ is that it equals to $G_{\mu \nu}$ on the self-accelerated solution given below, while it contributes in a non-trivial way to perturbations about it.

The action functional that gives rise to this new set of equations happens to be the one that we have already discussed in the previous section (35). The only difference is that in the junction condition (39) we need to flip the sign of the coefficient in front of the extrinsic curvature terms. This can be achieved if we choose $\bar{M}>M_{*}$ and set

$$
m_{c} \equiv \frac{2\left(\bar{M}^{3}-M_{*}^{3}\right)}{M_{\mathrm{Pl}}^{2}} .
$$

The metric for the self-accelerated solution of the new system of equations (46, 6-8) reads:

$$
P(t, y)=1-|y| \frac{\ddot{a}}{\sqrt{\dot{a}^{2}+k}}, \quad Q(t, y)=a(t)-|y| \sqrt{\dot{a}^{2}+k}, \quad \Sigma(t, y)=1,
$$

where we have chosen a negative sign in front of the terms proportional to $|y|$. Let us now see how the change of the positive signs in the metric (41) into the negative signs in (48) changes the value of the extrinsic curvature evaluated at $y=0^{+}$. On the solution (48), $N_{\mu}=0, N=1$, and $K_{\mu \nu}=\partial_{y} g_{\mu \nu} / 2$. Hence, at $y=0^{+}$the components of the extrinsic curvature tensor evaluated on the solution (48) equal to minus the corresponding components evaluated on (41). Therefore, substitution of (41) into (3) would give the same equation as the substitution of (48) into (46). The corresponding Friedmann equation on the empty brane, which now follows from (46)) instead of (3), reads:

$$
H^{2}+\frac{k}{a^{2}}=m_{c} \sqrt{H^{2}+\frac{k}{a^{2}}} .
$$

For $k=0$ this coincides with (42) and gives the spatially-flat dS solution with $H=m_{c}$ (45). For general $k$ the solutions are:

$$
d s^{2}=(1-H|y|)^{2}\left\{-d t^{2}+a^{2}(t)\left(d \chi^{2}+S_{k}^{2}(\chi) d \Omega^{2}\right)\right\}+d y^{2},
$$

where $H=m_{c}$ and $k=-1,0,1$ corresponds to the open, flat and closed spatial slicing of $4 \mathrm{D}$ dS space, for which $S_{k}(\chi)=\sinh \chi, \chi, \sin \chi$, respectively 9 .

The solution (50) should satisfy all the bulk equations (6] - 8), since in the bulk it is locally equivalent to Minkowski space. We checked by direct substitution that (50) solves Eqs. (6 - 8) too. The matter/radiation density can also be introduced as described above. The Freedman equation coincides with (43) with $\epsilon$ set to 1.

\footnotetext{
${ }^{9}$ There are two other solutions to (49). For $k=0$ one finds the $H=0$ flat solution. For $k=-1$ one finds the Milne solution $a(t)=t$.
} 
It still remains to be shown that the modified model described by (35) gets read of all the negative mass states that may appear in the self-accelerated solution, this question will be discussed in [53. I just point out that an additional benefit of the new term in (35) is that it allows to relax the constraint on the bulk gravity scale. The latter can take an arbitrary value below $M_{\mathrm{Pl}}$. This opens a window for a possible string theory realization of this model, or its $D>5$ counterparts [5, 7] (for earlier proposals see [51, 52]).

\section{Acknowledgments}

I would like to thank the organizers of the Cargèse 2007 School, especially Laurent Baulieu, Pierre Vanhove, Paul Windy, and Elena Gianolio. The work is supported by NASA grant NNGG05GH34G and NSF grant 0403005.

\section{References}

[1] A. G. Riess et al. [Supernova Search Team Collaboration], Astron. J. 116, 1009 (1998) arXiv:astro-ph/9805201;

S. Perlmutter et al. [Supernova Cosmology Project Collaboration], Astrophys. J. 517, 565 (1999) |arXiv:astro-ph/9812133].

[2] G. Dvali, G. Gabadadze and M. Porrati, Phys. Lett. B485, 208 (2000) hep-th/0005016.

[3] S. Weinberg, Rev. Mod. Phys. 61, 1 (1989).

[4] G. Gabadadze, arXiv:hep-th/0408118; In Ian Kogan Memorial Volume, Shifman, M. (ed.) et al. World Scientific, 2004; vol.2, pp 1061-1130.

[5] G. R. Dvali and G. Gabadadze, Phys. Rev. D 63, 065007 (2001) arXiv:hep-th/0008054.

[6] G. Dvali, G. Gabadadze and M. Shifman, Phys. Rev. D 67, 044020 (2003) arXiv:hep-th/0202174;

G. Dvali, G. Gabadadze and M. Shifman, arXiv:hep-th/0208096. Published in *Minneapolis 2002, Continuous advances in QCD* 566-581.

[7] G. Gabadadze and M. Shifman, Phys. Rev. D 69, 124032 (2004) arXiv:hep-th/0312289.

[8] R. Arnowitt, S. Deser and C. W. Misner, "Gravitation: an introduction to current research", L. Witten ed. (Wiley 1962), pp 227-265; arXiv:gr-qc/0405109.

[9] G. Gabadadze, arXiv:hep-th/0612213. 
[10] G. R. Dvali, G. Gabadadze, M. Kolanovic and F. Nitti, Phys. Rev. D 64, 084004 (2001) arXiv:hep-ph/0102216.

[11] H. van Dam and M. J. G. Veltman, Nucl. Phys. B 22, 397 (1970);

V. I. Zakharov, JETP Lett. 12 (1970) 312 [Pisma Zh. Eksp. Teor. Fiz. 12 (1970) 447].

[12] A. I. Vainshtein, Phys. Lett. B 39 (1972) 393.

[13] C. Deffayet, G. R. Dvali, G. Gabadadze and A. I. Vainshtein, Phys. Rev. D 65, 044026 (2002) arXiv:hep-th/0106001.

[14] A. Gruzinov, New Astron. 10, 311 (2005) arXiv:astro-ph/0112246.

[15] G. Gabadadze, Phys. Rev. D 70, 064005 (2004) arXiv:hep-th/0403161.

[16] C. Middleton and G. Siopsis, Phys. Lett. B 613, 189 (2005) arXiv:hep-th/0502020].

[17] M. Porrati and J. W. Rombouts, Phys. Rev. D 69, 122003 (2004) arXiv:hep-th/0401211.

[18] M. N. Smolyakov, Phys. Rev. D 72, 084010 (2005) arXiv:hep-th/0506020].

[19] C. Deffayet, Phys. Lett. B 502, 199 (2001) arXiv:hep-th/0010186.

[20] C. Deffayet, G. R. Dvali and G. Gabadadze, Phys. Rev. D 65, 044023 (2002) astro-ph/0105068.

[21] C. Deffayet, S. J. Landau, J. Raux, M. Zaldarriaga and P. Astier, Phys. Rev. D 66, 024019 (2002) arXiv:astro-ph/0201164.

[22] A. Lue, R. Scoccimarro and G. Starkman, Phys. Rev. D 69, 044005 (2004) arXiv:astro-ph/0307034. Phys. Rev. D 69, 124015 (2004).

[23] D. Jain, A. Dev and J. S. Alcaniz, Phys. Rev. D 66, 083511 (2002) arXiv:astro-ph/0206224. J. S. Alcaniz, D. Jain and A. Dev, Phys. Rev. D 66, 067301 (2002).

[24] M. Ishak, A. Upadhye and D. N. Spergel, Phys. Rev. D 74, 043513 (2006) arXiv:astro-ph/0507184.

[25] E. V. Linder, Phys. Rev. D 72, 043529 (2005) arXiv:astro-ph/0507263.

[26] L. Knox, Y. S. Song and J. A. Tyson, arXiv:astro-ph/0503644.

[27] R. Maartens and E. Majerotto, Phys. Rev. D 74, 023004 (2006) arXiv:astro-ph/0603353. 
[28] K. Koyama and R. Maartens, JCAP 0601, $016 \quad$ (2006) arXiv:astro-ph/0511634.

[29] I. Sawicki and S. M. Carroll, arXiv:astro-ph/0510364.

[30] Y. S. Song, I. Sawicki and W. Hu, arXiv:astro-ph/0606286.

[31] T. Tanaka, Phys. Rev. D 69, 024001 (2004) arXiv:gr-qc/0305031.

[32] G. Gabadadze and A. Iglesias, Phys. Rev. D 72, 084024 (2005) arXiv:hep-th/0407049;

[33] G. Dvali, A. Gruzinov and M. Zaldarriaga, Phys. Rev. D 68, 024012 (2003) arXiv:hep-ph/0212069.

[34] A. Lue and G. Starkman, Phys. Rev. D 67, 064002 (2003) arXiv:astro-ph/0212083.

[35] L. Iorio, Class. Quant. Grav. 22, 5271 (2005) arXiv:gr-qc/0504053]; JCAP 0509, 006 (2005) |arXiv:gr-qc/0508047]; JCAP 0601, 008 (2006).

[36] G. Gabadadze and A. Iglesias, Phys. Lett. B 632, 617 (2006) arXiv:hep-th/0508201.

[37] J. G. Williams, X. X. Newhall and J. O. Dickey, Phys. Rev. D 53, 6730 (1996); J.G. Williams, D.H. Boggs, J.O. Dickey, W.M. Folkner, "Lunar tests of gravitational physics", Proceedings of the Ninth Marcel Grossman Meeting, Rome, Italy, June 2000, ed. R. Jantzen; World Scientific Co., (2001)

[38] T.W. Murphy, Jr., J.D. Strasburg, C.W. Stubbs, E.G. Adelberger, J. Angle, K. Nordtvedt, J.G. Williams, J.O. Dickey, B. Gillespie, "The Apace Point Observatory Lunar Laser-ranging Operation (APOLLO), "12th International Lunar Laser Ranging Workshop, Matera, Italy, 2000.

[39] G. Dvali, G. Gabadadze, X. r. Hou and E. Sefusatti, Phys. Rev. D 67, 044019 (2003) arXiv:hep-th/0111266.

[40] A. Lue and G. D. Starkman, Phys. Rev. D 70, 101501 (2004) arXiv:astro-ph/0408246.

[41] N. Arkani-Hamed, H. Georgi and M. D. Schwartz, Annals Phys. 305, 96 (2003) arXiv:hep-th/0210184.

[42] G. Gabadadze and A. Iglesias, Phys. Lett. B 639, 88 (2006) arXiv:hep-th/0603199.

[43] M. A. Luty, M. Porrati and R. Rattazzi, JHEP 0309, 029 (2003) arXiv:hep-th/0303116. 
[44] K. Koyama, Phys. Rev. D 72, 123511 (2005) arXiv:hep-th/0503191.

[45] D. Gorbunov, K. Koyama and S. Sibiryakov, Phys. Rev. D 73, 044016 (2006) arXiv:hep-th/0512097.

[46] C. Charmousis, R. Gregory, N. Kaloper and A. Padilla, JHEP 0610, 066 (2006) arXiv:hep-th/0604086].

[47] C. Deffayet, G. Gabadadze and A. Iglesias, JCAP 0608, 012 (2006) arXiv:hep-th/0607099.

[48] G. Dvali, arXiv:hep-th/0610013.

[49] G. Dvali, G. Gabadadze, O. Pujolas and R. Rahman, arXiv:hep-th/0612016.

[50] N. Kaloper and A. D. Linde, Phys. Rev. D 59, 101303 (1999) arXiv:hep-th/9811141.

[51] I. Antoniadis, R. Minasian and P. Vanhove, Nucl. Phys. B 648, 69 (2003) arXiv:hep-th/0209030.

[52] E. Kohlprath, Nucl. Phys. B 697, 243 (2004) arXiv:hep-th/0311251. E. Kohlprath and P. Vanhove, arXiv:hep-th/0409197.

[53] G. Gabadadze, in progress. 